\title{
Analytic Techniques for PDEs Fourier Series and the Method of Separation of Variables
}

Hend Elmhdi

Follow this and additional works at: https://researchrepository.wvu.edu/etd

\section{Recommended Citation}

Elmhdi, Hend, "Analytic Techniques for PDEs Fourier Series and the Method of Separation of Variables" (2017). Graduate Theses, Dissertations, and Problem Reports. 5540.

https://researchrepository.wvu.edu/etd/5540

This Thesis is protected by copyright and/or related rights. It has been brought to you by the The Research Repository @ WVU with permission from the rights-holder(s). You are free to use this Thesis in any way that is permitted by the copyright and related rights legislation that applies to your use. For other uses you must obtain permission from the rights-holder(s) directly, unless additional rights are indicated by a Creative Commons license in the record and/ or on the work itself. This Thesis has been accepted for inclusion in WVU Graduate Theses, Dissertations, and Problem Reports collection by an authorized administrator of The Research Repository @ WVU. For more information, please contact researchrepository@mail.wvu.edu. 
Analytic Techniques for PDEs Fourier Series and the

\author{
method of separation of variables \\ Hend Elmhdi
}

Thesis submitted to the Eberly College of Arts and Sciences at West Virginia University

in partial fulfillment of the requirements for the degree of Master of Science in Mathematics

Charis Tsikkou PhD Chair

Harvey Diamond PhD

Marjorie Darrah PhD

Department of Mathematics

Morgantown, West Virginia 2017

Keywords: Fourier Series Technique

Copyright 2017

Hend EImhdi 


\begin{abstract}
Analytic Techniques for PDEs Fourier Series and the method of separation of variables
\end{abstract}

\title{
Hend Elmhdi
}

The main topic of this study is deriving solutions of linear PDEs that have no mixed derivatives, using the method of separation of variables. One seeks a solution of a $P D E$, involving $n$ independent variables, in a form of a product or sum and converts the PDE into $n$ solvable ordinary differential equations to build the general solution out of these particular solutions. 


\section{Contans}

- Fourier Series

- The Computation of the usual Fourier series is based on the integral identities

(9)

- Functions of Period 2L

- Even and Odd functions, Half Range Expansions

- Harmonic Anyalysis

$\bullet$

- Orthogonal Series: Generalized Fourier series

$\bullet$

- Double Fourier Series

- Wave Equation

- Solution of the one Dimensional Wave equation

- Heat Equation

- Introduction to separation of variables and equation

-

- How we use Fourier Series

- The two Dimensional Wave and Heat Equations

- Solution of the separated Equations

- Double Fourier series solution of the entire problem 
- The solution of the two Dimensional wave equation

- Solution of the two Dimensional Heat equation for a Rectangle

- Laplace's Equation in Rectangular coordinates

- References 


\title{
Analytic Techniques for PDEs Fourier Series and the
}

\section{method of separation of variables \\ Hend Elmhdi}

\begin{abstract}
The main topic of this study is the solution of linear PDEs that have no mixed derivatives using the method of separation of variables. One seeks a solution of a PDE involving $\mathrm{n}$ independent variables in a form of product or sum, and converts the PDE into $\mathrm{n}$ ordinary differential equations to build the general solution out of these particular solutions.
\end{abstract}

\section{1-Fourier Series:}

We will consider Fourier series and how important in engineering and other sciences. Function $f$ can be represented by an infinite series of elementary trigonometric functions sines and cosines. We first need some background material. A function $f(x)$ is called a periodic function if $f(x)$ is defined for all real $x$ and $f(x)=f(x+P)$ for all $x$.

$P$ is called the period of $f(x)$.

Example1: we have $\sin (x+2 \pi)=\sin x$ for all $x$, therefore $P=2 \pi$

Example2: $f(x)=\cot (x), \quad g(x)=\tan (x)$ have period $\pi$ since $\tan (x+\pi)=\operatorname{tax}(x), \cot (x+\pi)=\cot (x)$ for all $x$ 
We will consider the Fourier series and how the representation of some of the functions that are important in engineering sciences and other sciences Fourier series. We first need some background material. A function $f(x)$ is called a periodic function if $f(x)$ is defined for all real $\mathrm{x}$. if we have a function $f(x)$, the function $f(x)$ called the function of a cycle, then we have $f(x \mp p)$

Ex 1: $f(x)=\sin (x)$, If $T=2 \pi$ then for every $x$ we have $\sin (x+2 \pi)=\sin (x)$

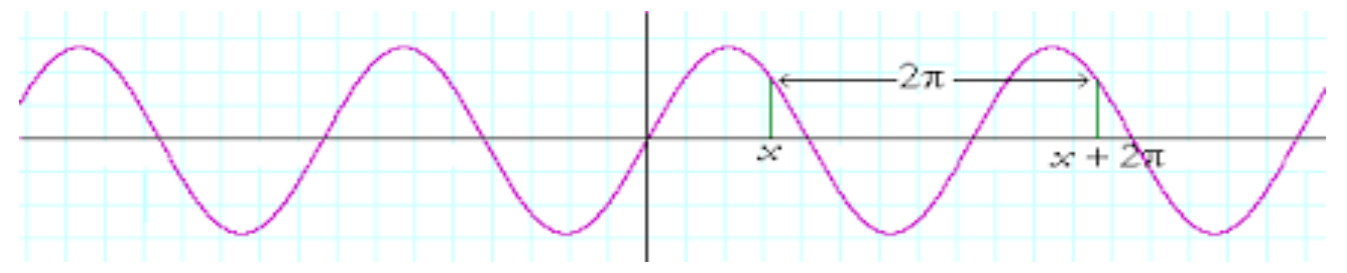

Figure $1 \sin x$

Ex2: $f(x)=\cot (x), f(x)=\tan (x)$ then $\tan (x+\pi)=\tan (x), \cot (x+\pi)=\cot (x)$

Notes:

1- If $f(x)$ is a periodic function for T, then $f(x \pm n T)=f(x)$ for every $n \in N$

$\int_{0}^{T} f(x) d x=\int_{c}^{c+T} f(x) d x$ for every $c \in N$

Then $f(x)$ has period $p$ it also has period $2 p$

$f(x+2 p)=f([x+p]+p)=f(x+p)=f(x)$ then

$f(x+n p)=f(x)$, for all $x$

2- If $f(x)$ and $g(x)$ have period $p$, then $a f(x)+b g(x)$ with any constants $a$ and $b$ also has the period $p$.

3- Simple function: 


$\begin{array}{lc}\cos x & \sin x \\ \cos 2 x & \sin 2 x \\ -- & -- \\ \cos n x & \operatorname{sinn} x\end{array}$

All these functions have period $2 \pi, \pi=3.14$. The form is called trigonometric system.
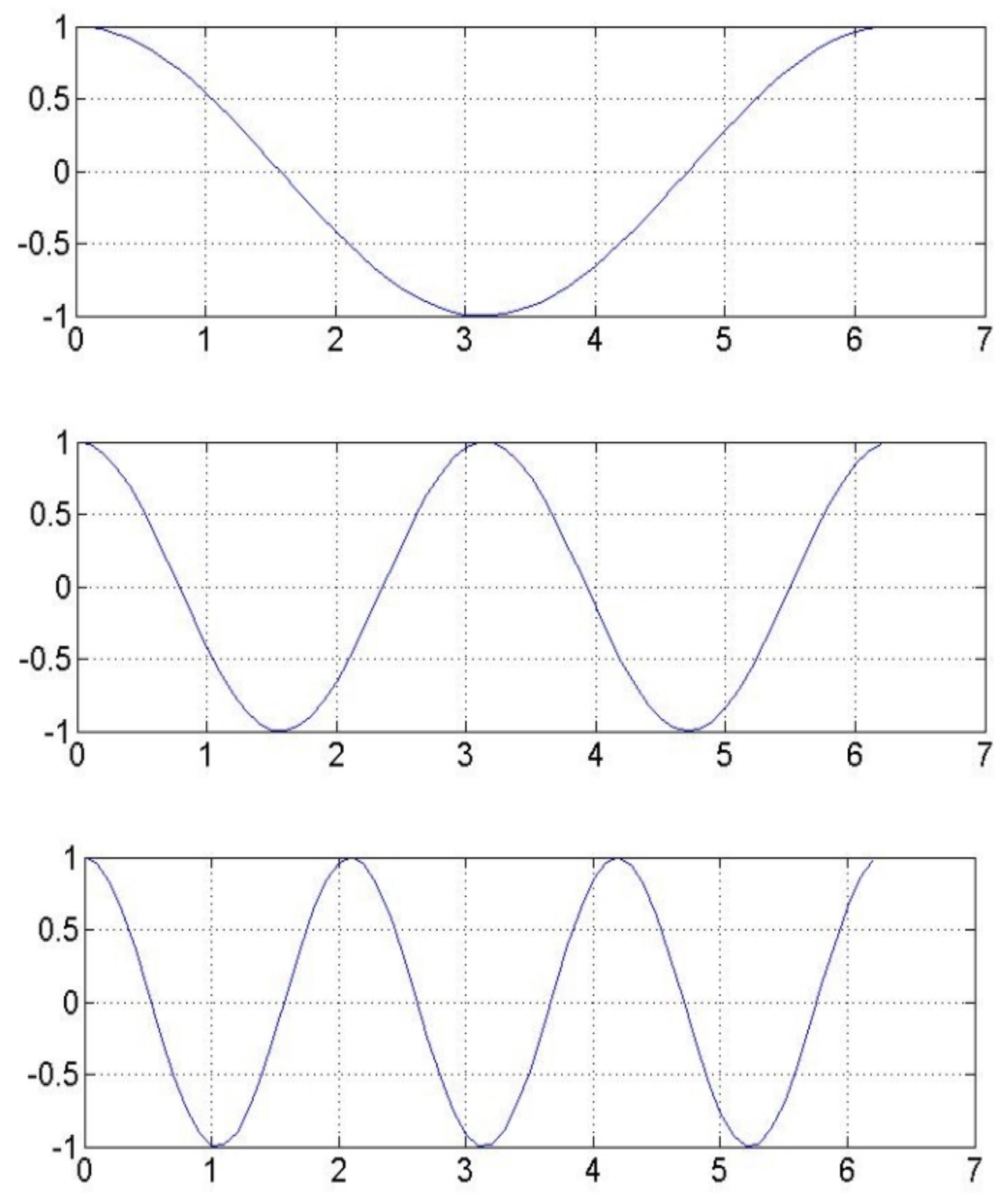

Figure $2 \sin x, \sin 52 x, \sin 3 x$ 

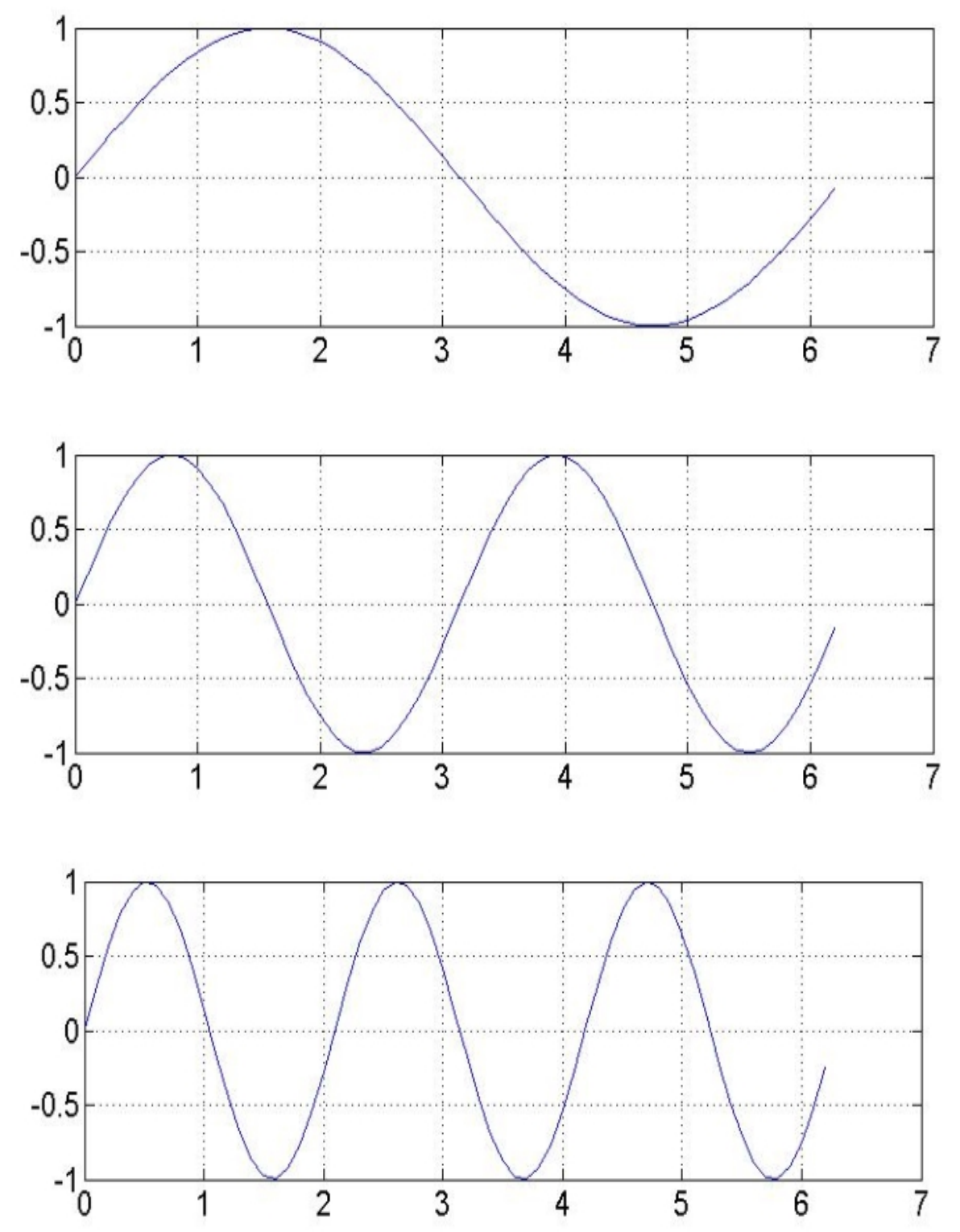

Figure $3 \cos x, \cos 2 x, \cos 3 x$

Cosine and Sine functions have the period $2 \pi$. Then we have now

$$
\begin{array}{r}
f(x)=a_{0}+a_{1} \cos x+b_{1} \sin x+a_{2} \cos 2 x+b_{2} \sin 2 x \pm--- \\
\boldsymbol{f}(\boldsymbol{x})=\boldsymbol{a}_{\mathbf{0}}+\sum_{\boldsymbol{n}=\mathbf{1}}^{\infty}\left(\boldsymbol{a}_{\boldsymbol{n}} \cos \boldsymbol{n} \boldsymbol{x}+\boldsymbol{b}_{\boldsymbol{n}} \sin \boldsymbol{n} \boldsymbol{x}\right)
\end{array}
$$


$a_{0}, a_{1}, b_{1}, a_{2}, b_{2}----$ are constants called coefficients of the series and each them has the period $2 \pi$. Suppose $f(x)$ dis a given function of period of $2 \pi$ then using the equality sign $f(x)=a_{0}+\sum_{n=1}^{\infty}\left(a_{n} \cos n x+b_{n} \sin n x\right)$ this is called the Fourier Series of $f(x)$ the coefficients of this series are called Fourier Coefficients of $\boldsymbol{f}(\boldsymbol{x})$

$$
\begin{aligned}
& a_{0}=\frac{1}{2 \pi} \int_{-\pi}^{\pi} f(x) d x \\
& a_{n}=\frac{1}{\pi} \int_{-\pi}^{\pi} f(x) \cos n x d x \quad n=1,2, \ldots . \\
& b_{n}=\frac{1}{\pi} \int_{-\pi}^{\pi} f(x) \sin (n x) d x \quad n=1,2, \ldots . .
\end{aligned}
$$

\section{Basics Examples:}

\section{Ex. 1: Find the Fourier series expansion of the periodic function}

$f(x)=x, \quad-\pi \leq x \leq \pi, f(x+2 \pi)=f(x)$

Solution: $a_{0}=\frac{1}{2 \pi} \int_{-\pi}^{\pi} x d x=0 \quad a_{n}=\frac{1}{\pi} \int_{-\pi}^{\pi} x \cos (n x) d x=0$

$$
b_{n}=\frac{1}{\pi} \int_{-\pi}^{\pi} x \sin (n x) d x=\frac{2}{\pi} \int_{0}^{\pi} x \sin (n x) d x=\frac{2}{n}(-1)^{n+1}
$$

Therefore, the expansion of the given function on $[-\pi, \pi]$ is given by

$$
x=2\left[\sin x-\frac{\sin (2 x)}{2}+\frac{\sin (3 x)}{3}-\frac{\sin (4 x)}{4}+\cdots\right]
$$

Ex. 2: Find the Fourier coefficients of the periodic function $f(x)$

$$
f(x)=\left\{\begin{array}{rl}
-k & \text { if }-\pi<x<0 \\
k & \text { if } 0<x<\pi
\end{array} \quad f(x+2 \pi)=f(x)\right.
$$

Solution: $a_{0}=0 \quad$ the area under the curve of $f(x)$ between $-\pi$ and $\pi$ is 0 


$$
\begin{aligned}
& a_{n}=\frac{1}{\pi} \int_{-\pi}^{\pi} f(x) \cos (n x) d x=\frac{1}{\pi}\left[\int_{-\pi}^{0}-k \cos (n x) d x+\right. \\
& \left.\int_{0}^{\pi} k \cos (n x) d x\right]=\frac{1}{\pi}\left[-\left.\frac{k \sin (n x)}{n}\right|_{-\pi} ^{0}+\left.\frac{k \sin (n x)}{n}\right|_{0} ^{\pi}\right]=0 \text { because } \sin n x=0 \text { at }-\pi, 0 \text { and }
\end{aligned}
$$

all these cosine coefficients are 0 . Then the Fourier series of $f(x)$ has no cosine terms, just sine terms, it is a Fourier Sin series with coefficients $b_{1}, b_{2}, \ldots \ldots$

$$
\begin{aligned}
& b_{n}=\frac{1}{\pi} \int_{-\pi}^{\pi} f(x) \sin (n x) d x=\frac{1}{\pi}\left[\int_{-\pi}^{0}-k \sin (n x) d x+\right. \\
& \left.\int_{0}^{\pi} k \sin (n x) d x\right]=\frac{1}{\pi}\left[-\left.\frac{k \cos (n x)}{n}\right|_{-\pi} ^{0}+\left.\frac{k \cos (n x)}{n}\right|_{0} ^{\pi}\right]
\end{aligned}
$$

We know $\cos (-\alpha)=\cos (\alpha)$ and $\cos (0)=1$

$$
b_{n}=\frac{k}{n \pi}[\cos 0-\cos (-n \pi)-\cos (n \pi)-\cos 0]=\frac{2 k}{n \pi}[1-\cos (n \pi)]
$$

Now $\cos (\pi)=-1, \cos (2 \pi)=1, \cos (3 \pi)=-1$,

$$
\cos (n \pi)=\left\{\begin{array}{ll}
-1 & \text { for odd } n \\
+1 & \text { for even } n
\end{array}\right. \text { and }
$$

$1-\cos (n \pi)=\left\{\begin{array}{lr}2 & \text { for odd } n \\ 0 & \text { for even } n\end{array}\right.$

The Fourier coefficients $b_{n}$ of our function are:

$$
b_{1}=\frac{4 k}{\pi}, b_{2}=0, b_{3}=\frac{4 k}{3 \pi}, \quad b_{4}=0, b_{5}=\frac{4 k}{5 \pi}, \ldots \ldots
$$

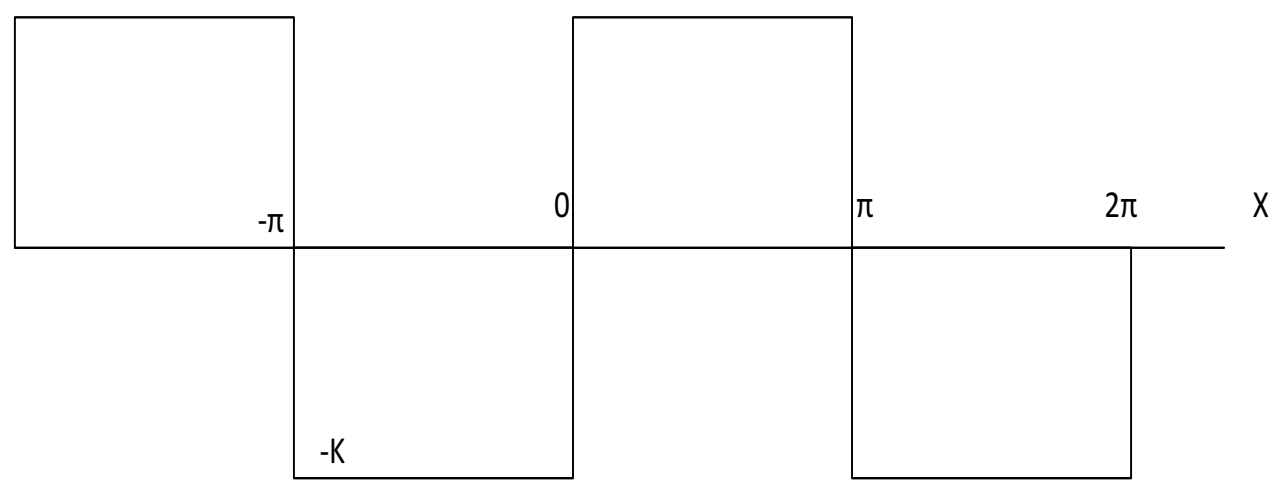


Since $a_{n}$ are 0 , the Fourier series of $f(x)$ is

$\frac{4 k}{\pi}\left(\sin x+\frac{1}{3} \sin 3 x+\frac{1}{5} \sin 5 x+\ldots ..\right)$

Ex. 3: Find the Fourier coefficients of the periodic function $f(x)$

$$
\begin{aligned}
& f(x)=x^{2} \quad 0<x<2, \quad 2 T=2, \quad T=1 \\
& a_{0}=\frac{1}{2 T} \int_{0}^{2 T} f(x) d x=\frac{1}{2.1} \int_{0}^{2 T} x^{2} d x=\left.\frac{x^{3}}{2.3}\right|_{0} ^{2}=\frac{8}{2.3} \\
& a_{n}=\frac{1}{T} \int_{0}^{2 T} f(x) \cos \left(\frac{n \pi}{T} x\right) d x=1 \int_{0}^{2} x^{2} \cos \left(\frac{n \pi}{1} x\right) d x \\
& a_{n}=\left.\left(x^{2} \frac{\sin (n \pi x)}{n \pi}+2 x \frac{-\cos (n \pi x)}{n^{2} \pi^{2}}+2 \frac{-\sin (n \pi x)}{n^{3} \pi^{3}}\right)\right|_{0} ^{2} \\
& a_{n}=\left.\left(x^{2} \frac{\sin (0)}{n \pi}+2 x \frac{-\cos (0)}{n^{2} \pi^{2}}+2 \frac{-\sin (n \pi x)}{n^{3} \pi^{3}}\right)\right|_{0} ^{2}=4 \frac{\cos (2 n \pi)}{n^{2} \pi^{2}}=\frac{4}{n^{2} \pi^{2}} \\
& b_{n}=\frac{1}{T} \int_{0}^{2 T} f(x) \sin \left(\frac{n \pi}{T} x\right) d x=1 \int_{0}^{2} x^{2} \sin \left(\frac{n \pi}{1} x\right) d x \\
& b_{n}=\left.\left(x^{2} \frac{-\cos (n \pi x)}{n \pi}-2 x \frac{-\sin (n \pi x)}{n^{2} \pi^{2}}+2 \frac{\cos (n \pi x)}{n^{3} \pi^{3}}\right)\right|_{0} ^{2} \\
& b_{n}=\left(\frac{-4 \cos (2 n \pi)}{n \pi}+2 \frac{\cos (n \pi x)}{n^{3} \pi^{3}}-\frac{-2}{n^{3} \pi^{3}}\right)=\frac{-4}{n \pi} \\
& f(x)=\frac{8 / 3}{2}+\sum_{n=1}^{\infty}\left(\frac{4}{n^{2} \pi^{2}} \cos n \pi x-\frac{-4}{n \pi} \sin n \pi x\right)
\end{aligned}
$$

1.1 Theorem: The Computation of the usual Fourier series is based on the integral identities:

a) $\int_{-\pi}^{\pi} \cos (n x) \cos (m x) d x=0(n \neq m)$

b) $\int_{-\pi}^{\pi} \sin (n x) \sin (m x) d x=0(n \neq m)$

c) $\int_{-\pi}^{\pi} \sin (n x) \cos (m x) d x=0(n \neq m$ or $n=m)$ 
Proof: $\int_{-\pi}^{\pi} \cos (n x) \cos (m x) d x=\frac{1}{2} \int_{-\pi}^{\pi} \cos (n+m) x d x+\frac{1}{2} \int_{-\pi}^{\pi} \cos (n-m) x d x=$

$\int_{-\pi}^{\pi} \sin (n x) \sin (m x) d x=\frac{1}{2} \int_{-\pi}^{\pi} \cos (n-m) x d x-\frac{1}{2} \int_{-\pi}^{\pi} \cos (n+m) x d x$

Since $m \neq n$

$\int_{-\pi}^{\pi} \sin (n x) \cos (m x) d x=\frac{1}{2} \int_{-\pi}^{\pi} \sin (n+m) x d x+\frac{1}{2} \int_{-\pi}^{\pi} \sin (n-m) x d x=0+0$

Using equations (1) (2) (3), we have

$\int_{-\pi}^{\pi} f(x) d x=\int_{-\pi}^{\pi}\left[a_{0}+\sum_{n=1}^{\infty}\left(a_{n} \cos n x+b_{n} \sin n x\right)\right] d x=a_{0} .2 \pi$

$\int_{-\pi}^{\pi} f(x) d x=a_{0} \int_{-\pi}^{\pi} d x+\sum_{n=1}^{\infty}\left(a_{n} \int_{-\pi}^{\pi} \cos n x d x+b_{n} \int_{-\pi}^{\pi} \sin n x d x\right)$

Then:

$\int_{-\pi}^{\pi} f(x) \operatorname{cosm} x d x=\int_{-\pi}^{\pi}\left[a_{0}+\sum_{n=1}^{\infty}\left(a_{n} \cos n x+b_{n} \sin n x\right)\right] \operatorname{cosm} x d x=a_{m} \int_{-\pi}^{\pi} \cos ^{2}(m x) d x$
$\int_{-\pi}^{\pi} f(x) \operatorname{sinm} x d x=\int_{-\pi}^{\pi}\left[a_{0}+\sum_{n=1}^{\infty}\left(a_{n} \cos n x+b_{n} \sin n x\right)\right] \operatorname{sinm} x d x=b_{m} \int_{-\pi}^{\pi} \sin ^{2}(m x) d x$

\subsection{Functions of Period 2L}

For a function $f(x)$ periodic on an interval $[-L, L]$ instead of $[-\pi, \pi]$ a simple change of variables can be used to $x=\frac{\pi \dot{x}}{L}, d x=\frac{\pi d \dot{x}}{L}$

Solving for $\dot{x}^{\prime}$ gives $\dot{x}=\frac{L x}{\pi}$ and plugging this in gives: 
$f(\dot{x})=a_{0}+\sum_{n=1}^{\infty}\left(a_{n} \cos \frac{n \pi \dot{x}}{L}+\sum_{n=1}^{\infty} b_{n} \sin \frac{n \pi \dot{x}}{L}\right.$

Therefore; $a_{0}=\frac{1}{2 L} \int_{-L}^{L} f(\dot{x}) d \dot{x} \quad a_{n}=\frac{1}{L} \int_{-L}^{L} f(\dot{x}) \cos \left(\frac{n \pi \dot{x}}{L}\right) d \dot{x} \quad b_{n}=\frac{1}{L} \int_{-L}^{L} f(\dot{x}) \sin \left(\frac{n \pi \dot{x}}{L}\right) d \dot{x}$

Ex: The function is instead defined on the interval $[0,2 L]$ then:

$$
\begin{aligned}
& a_{0}=\frac{1}{2 L} \int_{0}^{2 L} f(\dot{x}) d \dot{x} \\
& a_{n}=\frac{1}{L} \int_{0}^{2 L} f(\dot{x}) \cos \left(\frac{n \pi \dot{x}}{L}\right) d \dot{x} \\
& b_{n}=\frac{1}{L} \int_{-L}^{L} f(\dot{x}) \sin \left(\frac{n \pi \dot{x}}{L}\right) d \dot{x}
\end{aligned}
$$

\subsection{Even and Odd functions, Half Range Expansions:}

If a function is even so that $f(x)=f(-x)$ then $f(x) \sin (n x)$ is odd. Therefore, $b_{n}=0$ for all $\mathrm{n}$

Similarly, if a function is odd so that $f(x)=-f(-x)$ then $f(x) \cos (n x)$ is odd. Therefore, $a_{n}=0$ for all $\mathrm{n}$

\begin{tabular}{|l|l|}
\hline Even function & Odd function \\
\hline Cosine harmonic & Sine harmonic \\
\hline$b_{n}=0$ & $a_{0}=0$, \\
& $a_{n}=0$ \\
\hline$a_{0}=\frac{1}{\pi} \int_{0}^{\pi} F(x) d x$ & $b_{n}=\frac{2}{\pi} \int_{0}^{\pi} F(x) \sin (n x) d x$ \\
\hline$a_{n}=\frac{2}{\pi} \int_{0}^{\pi} F(x) \cos (n x) d x$ & $F(x)=\sum_{n=1}^{\infty} b_{n} \sin (n x)$ \\
\hline
\end{tabular}




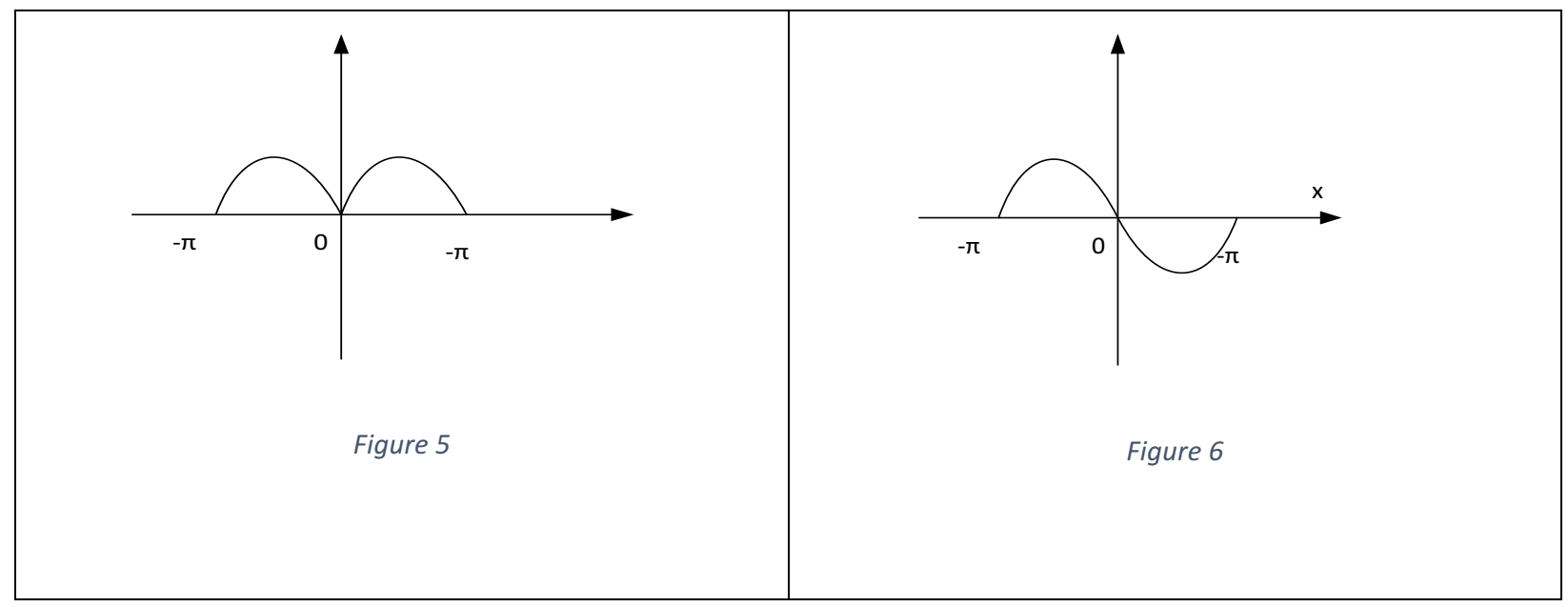

Notes:

\begin{tabular}{|l|l|}
\hline $\sin n \pi=0$ & $\cos (n \pi)=(-1)^{n}$ \\
\hline if $n=1 \rightarrow \sin 180=0$ & $\cos 180=-1$ \\
\hline if $n=2 \rightarrow \sin 360=0$ & $\cos 360=1$ \\
\hline $\sin 2 n \pi=0$ & $\cos 2 n \pi=1$ \\
\hline $\sin ((2 n \pm 1) \pi)=0$ & $\sin ((2 n \pm 1) \pi)=-1$ \\
\hline $\sin \left(\frac{(2 n-1) \pi}{2}\right)=(-1)^{n+1}$ & $\cos \left(\frac{(2 n-1) \pi}{2}\right)=0$ \\
\hline
\end{tabular}

Ex: Since $v=\frac{\pi}{L} x$ we have $\frac{\pi}{L} d x$ 


$$
\begin{aligned}
f(x)=a_{0} & +\sum_{n=1}^{\infty}\left(a_{n} \cos \frac{n \pi x}{L}+b_{n} \sin \frac{n \pi x}{L}\right) \\
a_{0} & =\frac{1}{2 L} \int_{-L}^{L} f(x) d x \\
a_{n} & =\frac{1}{L} \int_{-L}^{L} f(x) \cos \left(\frac{n \pi x}{L}\right) d x, \quad n=1,2, \ldots \\
b_{n} & =\frac{1}{L} \int_{-L}^{L} f(x) \sin \left(\frac{n \pi x}{L}\right) d x, \quad n=1,2, \ldots
\end{aligned}
$$

\section{Ex: Find the Fourier series of the function}

$$
f(x)=\left\{\begin{array}{lr}
0 \text { if }-2<x<-1 \\
k \text { if }-1<x<1 \\
0 \text { if } 1<x<2 & p=2 L=4
\end{array}\right.
$$

Solution: $a_{0}=\frac{k}{2}$

$$
a_{n}=\frac{1}{2} \int_{-2}^{2} f(x) \cos \left(\frac{n \pi x}{2}\right) d x=\frac{1}{2} \int_{-1}^{1} \mathrm{k} \cos \left(\frac{n \pi x}{2}\right) d x=\frac{k}{n \pi} \sin \left(\frac{n \pi}{2}\right)
$$

Thus $a_{n}=0$ if $n$ is even and

$$
\begin{aligned}
& a_{n}=\frac{2 k}{n \pi} \text { if } n=1,5,9, \ldots . . \\
& a_{n}=\frac{-2 k}{n \pi} \text { if } n=3,7,11, \ldots .
\end{aligned}
$$

We have $b_{n}=0, n=1,2, \ldots$

$$
f(x)=\frac{k}{2}+\frac{2 k}{\pi}\left[\cos \left(\frac{\pi}{2} x\right)-\frac{1}{3} \cos \left(\frac{3 \pi}{2} x\right)+\frac{1}{5} \cos \left(\frac{5 \pi}{2} x\right)-\cdots+\cdots\right]
$$

\section{Ex: Find the Fourier series function}

$f(x)=\left\{\begin{array}{cl}-k & \text { if }-2<x<0 \\ k & \text { if } 0<x<2\end{array} \quad p=2 L=4, \quad L=2\right.$ 
Solution: Since $L=2$ we have $v=\frac{\pi x}{2}$

$g(v)=\frac{4 k}{\pi}\left[\sin v+\frac{1}{3} \sin 3 v+\frac{1}{5} \sin 5 v+\cdots\right]$

$f(x)=\frac{4 k}{\pi}\left[\sin \frac{\pi x}{2}+\frac{1}{3} \sin \frac{3 \pi x}{2}+\frac{1}{5} \sin 5 \frac{\pi x}{2}+\cdots\right]$

1.4 Harmonic Analysis:

$f(x)=\frac{a_{0}}{2}+a_{1} \cos x+b_{1} \sin x+a_{2} \cos 2 x+b_{2} \sin 2 x+\cdots$

$$
\begin{aligned}
& a_{0}=\frac{1}{2 \pi} \int_{0}^{2 \pi} f(x) d x=2 \cdot \frac{1}{2 \cdot 2 \pi} \int_{0}^{2 \pi} f(x) d x \\
& a_{n}=\frac{1}{\pi} \int_{0}^{2 \pi} f(x) \cos n x d x \\
& b_{n}=\frac{1}{\pi} \int_{0}^{2 \pi} f(x) \sin n x d x \\
& f(x)=a_{0}+a_{1} \cos x+b_{1} \sin x \\
& f(x)=a_{0}+a_{1} \cos x+b_{1} \sin x+a_{2} \cos 2 x+b_{2} \sin 2 x
\end{aligned}
$$

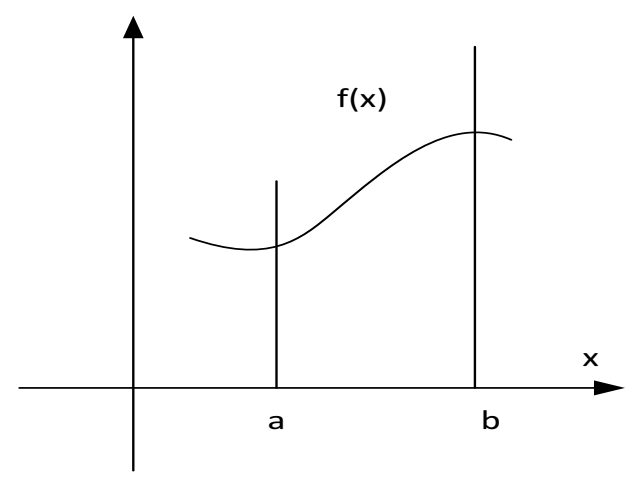

Figure 7

\section{Simplifications: even and odd functions}

If $f(x)$ is an even function that is $f(-x)=f(x)$ then it is Fourier cosine series 


$$
\begin{aligned}
& f(x)=a_{0}+\sum_{n=1}^{\infty} a_{n} \cos \left(\frac{n \pi x}{L}\right) \quad \text { if even } \\
& a_{0}=\frac{1}{L} \int_{0}^{L} f(x) d x \\
& a_{n}=\frac{2}{L} \int_{0}^{L} f(x) \cos \left(\frac{n \pi x}{L}\right) d x \quad n=1,2,3, \ldots \\
& f(x)=\sum_{n=1}^{\infty} b_{n} \sin \left(\frac{n \pi x}{L}\right) \quad \text { if odd } \\
& b_{n}=\frac{2}{L} \int_{0}^{L} f(x) \sin \left(\frac{n \pi x}{L}\right) d x \quad n=1,2,3, \ldots
\end{aligned}
$$

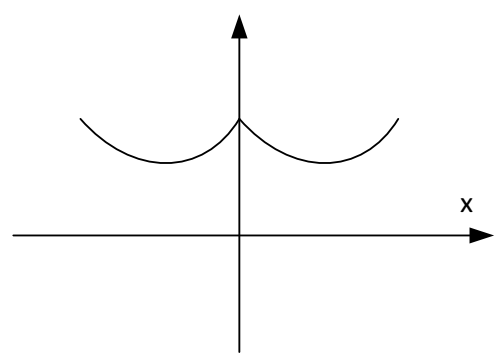

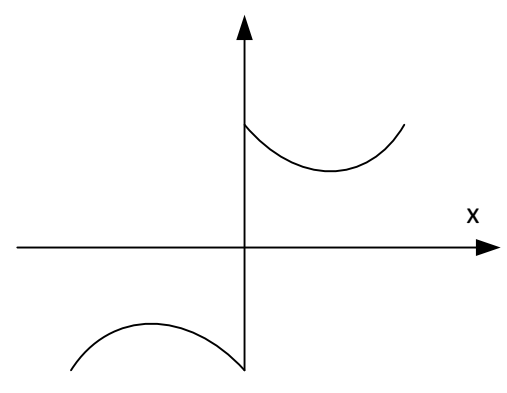

Figure 9 
We have

$\int_{-L}^{L} g(x) d x=2 \int_{0}^{L} g(x) d x \quad$ for even $g$

$\int_{-L}^{L} h(x) d x=0 \quad$ for odd $\mathrm{h}$

Then

*ven function of period of $2 \pi$

If $f$ is even and $L=\pi$, then

$$
\begin{aligned}
& f(x)=a_{0}+\sum_{n=1}^{\infty} a_{n} \cos (n x) \\
& a_{0}=\frac{1}{\pi} \int_{0}^{\pi} f(x) d x \quad a_{n}=\frac{2}{\pi} \int_{0}^{\pi} f(x) \cos n x d x \quad n=1,2,3, \ldots
\end{aligned}
$$

* Even function of period of $2 \pi$

If $f$ is odd and $L=\pi$, then

$$
\begin{aligned}
& f(x)=\sum_{n=1}^{\infty} b_{n} \sin (n x) \\
& b_{n}=\frac{2}{\pi} \int_{0}^{\pi} f(x) \sin n x d x \quad n=1,2,3, \ldots
\end{aligned}
$$

\section{Ex. Find the Fourier series of the function}

$$
\begin{aligned}
& f(x)=x+\pi \quad \text { if }-\pi<x<\pi \text { and } \\
& f(x+2 \pi)=f(x)
\end{aligned}
$$

Solution: $f=f_{1}+f_{2} \quad f_{1}=x, f_{2}=\pi$

$f_{1}$ is odd $a_{n}=0$ for $n=1,2,3, \ldots$ 
$b_{n}=\frac{2}{\pi} \int_{0}^{\pi} f_{1}(x) \operatorname{sinn} x d x=\frac{2}{\pi} \int_{0}^{\pi} x \operatorname{sinn} x d x=\frac{2}{\pi}\left[-\left.\frac{x \cos n x}{n}\right|_{0} ^{\pi}+\frac{1}{n} \int_{0}^{\pi} \cos n x d x\right]$

$b_{n}=\frac{2}{n} \cos n \pi$

Hence $b_{1}=2, \quad b_{2}=-\frac{2}{2}, \quad b_{3}=\frac{2}{3}, b_{4}=-\frac{2}{4}, \ldots$

The Fourier series of $f(x)$ is

$$
f(x)=\pi+2\left(\sin x-\frac{1}{2} \sin 2 x+\frac{1}{3} \sin 3 x-\cdots+\cdots\right)
$$

\section{Half- Range Expansions}

\section{Ex. Find the two half-range expansions of the function}

$$
f(x)=\left\{\begin{array}{cc}
\frac{2 k}{L} x \text { if } & 0<x<\frac{L}{2} \\
\frac{2 k}{L}(L-x) & \text { if } \frac{L}{2}<x<L
\end{array}\right.
$$

Solution:

even periodic extension

$$
\begin{aligned}
& a_{0}=\frac{1}{L}\left[\frac{2 k}{L} \int_{0}^{\frac{l}{2}} x d x+\frac{2 k}{L} \int_{\frac{L}{2}}^{L}(L-x) d x\right]=\frac{k}{2} \\
& a_{n}=\frac{2}{L}\left[\frac{2 k}{L} \int_{0}^{\frac{l}{2}} x \cos \left(\frac{n \pi}{L} x\right) d x+\frac{2 k}{L} \int_{\frac{L}{2}}^{L}(L-x) \cos \left(\frac{n \pi}{L} x\right) d x\right] \text { then } \\
& \int_{0}^{\frac{l}{2}} x \cos \left(\frac{n \pi}{L} x\right) d x=\left[\left.\frac{L x \sin \left(\frac{n x}{L} x\right)}{n \pi}\right|_{0} ^{\frac{L}{2}}-\frac{L}{n \pi} \int_{0}^{\frac{L}{2}} \sin \left(\frac{n \pi}{L} x\right) d x\right]= \\
& \frac{L^{2}}{2 \mathrm{n} \pi} \sin \left(\frac{n \pi}{2}\right)+\frac{L^{2}}{\pi^{2} n^{2}}\left(\cos \frac{n \pi}{2}-1\right)
\end{aligned}
$$




$$
\begin{aligned}
& \left.\int_{\frac{L}{2}}^{L}(L-x) \cos \left(\frac{n \pi}{L} x\right) d x\right]=\left[\left.\frac{L(L-x) \sin \left(\frac{n x}{L} x\right)}{n \pi}\right|_{L / 2} ^{L}+\frac{L}{n \pi} \int_{L / 2}^{L} \sin \left(\frac{n \pi}{L} x\right) d x\right]= \\
& \left(0-\frac{L}{2 n \pi}\left(L-\frac{L}{2}\right) \sin \frac{n \pi}{2}\right)-\frac{L^{2}}{\pi^{2} n^{2}}\left(\cos n \pi-\cos \frac{n \pi}{2}\right) \\
& a_{n}=\frac{4 k}{\pi^{2} n^{2}}\left(2 \cos \frac{n \pi}{2}-\cos n \pi-1\right) \\
& a_{2}=-\frac{16 k}{12^{2} \pi^{2}}, \quad a_{6}=-\frac{16 k}{16^{2} \pi^{2}}, \quad a_{10}=-\frac{16 k}{110^{2} \pi^{2}}, \ldots \ldots \\
& \text { And } a_{n}=0 \quad \text { if } n \neq 2,6,10,14 \ldots \ldots \\
& f(x)=\frac{k}{2}+\frac{16 k}{\pi^{2}}\left(\frac{1}{2^{2}} \cos \frac{2 \pi}{L} x+\frac{1}{6^{2}} \cos \frac{6 \pi}{L} x+\cdots\right)
\end{aligned}
$$

* Odd periodic extension

$$
\begin{aligned}
& b_{n}=\frac{8 k}{\pi^{2} n^{2}} \sin \frac{n \pi}{2} \\
& f(x)=\frac{8 k}{\pi^{2}}\left(\frac{1}{1^{2}} \sin \frac{\pi}{L} x-\frac{1}{3^{2}} \sin \frac{3 \pi}{L} x+\frac{1}{5^{2}} \sin \frac{5 \pi}{L} x \ldots\right)
\end{aligned}
$$

\subsection{Orthogonal Series: Generalized Fourier series}

Let $y_{0}, y_{1}, y_{2}, \ldots$. Be the orthogonal with respect to a weight function $r(x)$ on an interval $a \leq x$ and let $f(x)$ be a function that can be represented by a convergent series

$f(x)=\sum_{m=0}^{\infty} a_{m} y_{m}(x)=a_{0} y_{0}(x)+a_{1} y_{1}(x)+\cdots$

This is called an orthogonal series or generalized Fourier series.

Assume that integration is permissible 


$$
\begin{aligned}
& \left(f, y_{n}\right)=\int_{a}^{b} r f y_{n} d x=\int_{a}^{b} r\left(\sum_{m=0}^{\infty} a_{m} y_{m}\right) y_{n} d x=\sum_{m=0}^{\infty} a_{m} \int_{a}^{b} r y_{m} y_{n} d x=\sum_{m=0}^{\infty} a_{m}\left(y_{m}, y_{n}\right) \\
& a_{n}\left(y_{n}, y_{n}\right)=a_{n}\|y\|^{2} \text { thus }\left(f, y_{n}\right)=a_{n}\|y\|^{2} \\
& a_{m}=\frac{\left(f, y_{m}\right)}{\left\|y_{m}\right\|^{2}}=\frac{1}{\left\|y_{m}\right\|^{2}} \int_{a}^{b} r(x) f(x) y_{m}(x) d x \quad m=0,1,2, \ldots
\end{aligned}
$$

\section{Example1: Fourier Legendre Series:}

A Fourier Legendre series is an Eigen function expansion

$$
\begin{aligned}
& f(x)=\sum a_{m} p_{m}(x)=a_{0} p_{0}+a_{1} p_{1}(x)+\cdots=a_{0}+a_{1} x+a_{1}\left(\frac{3}{2} x^{2}-\frac{1}{2}\right)+\cdots \\
& a_{m}=\frac{2 m+1}{2} \int_{-1}^{1} f(x) p_{m}(x) d x \quad m=0,1,2, \ldots \\
& \left\|p_{m}\right\|=\sqrt{\int_{-1}^{1} p_{m}(x)^{2} d x}=\sqrt{\frac{2}{2 m+1}} \quad m=0,1,2, \ldots
\end{aligned}
$$

Ex: if $a_{m}=\frac{2 m+1}{2} \int_{-1}^{1} \sin \pi x p_{m}(x) d x$ thus:

$a_{m}=\frac{3}{2} \int_{-1}^{1} x \sin \pi x d x=\frac{3}{\pi}=0.95493$

Then the Fourier Legendre series of $\sin \pi x$ is

$\sin \pi x=0.95493 p_{1}(x)-1.15824 p_{3}(x)+021929 p_{5}(x)-0.01664 p_{7}(x)+\cdots$

\section{Example2: Fourier Bessel series}

For each fixed nonnegative integer $n$, the sequence of Bessel functions of the first kind

$$
J_{n}\left(K_{n, 1} x\right), J_{n}\left(K_{n, 2} x\right), \ldots \text { with } K_{n, m}, k k=\alpha_{n, m} \text { thus } k_{n, m}=\frac{\alpha_{n, m}}{R} \quad m=1,2, \ldots
$$


On the interval $0 \leq x \leq R$ with respect to the weight function $r(x)=x$ that is

$\int_{0}^{R} x J_{n}\left(K_{n, m} x\right) J_{n}\left(K_{n, j} x\right) d x=0 \quad(j \neq m, n$ fixed $)$

The orthogonal set for $J_{n}$ is $J_{n}\left(K_{n, 1} x\right), J_{n}\left(K_{n, 2} x\right), J_{n}\left(K_{n, 3} x\right), \ldots \quad \mathrm{n}$ is fixed and $K_{n, m}$ is given

$K R=\alpha_{n, m}, K_{n, m}=\frac{\alpha_{n, m}}{R}$

The Fourier Bessel series to $J_{n}$ is

$f(x)=\sum a_{m} J_{n}\left(K_{n, m} x\right)=a_{1} J_{n}\left(K_{n, 1} x\right)+a_{2} J_{n}\left(K_{n, 2} x\right)+\cdots$

$a_{m}=\frac{2}{R^{2} J_{n+1}^{2} \alpha_{n, m}} \int_{0}^{R} x f(x) J_{n}\left(K_{n, m} x\right) d x \quad m=0,1,2, \ldots$

$\left\|J_{n}\left(K_{n, m} x\right)\right\|^{2}=\int_{0}^{R} x J_{n}{ }^{2}\left(K_{n, m} x\right) d x=\frac{R^{2}}{2} J_{n+1}{ }^{2}\left(K_{n, m} R\right)$

\section{Example3: Fourier Bessel series}

Let $f(x)=1-x^{2} \quad R=1, \quad n=0$

$a_{m}=\frac{2}{J_{1}{ }^{2}(\lambda)} \int_{0}^{1} x\left(1-x^{2}\right) J_{0}(\lambda x) d x=\frac{2}{J_{1}{ }^{2}(\lambda)}\left[\left.\frac{1}{\lambda}\left(1-x^{2}\right) x J_{1}(\lambda x)\right|_{0} ^{1}-\frac{1}{\lambda} \int_{0}^{1} x(-2 x) J_{1}(\lambda x) d x\right]$

$a_{m}=\frac{4 J_{2}(\lambda)}{\lambda^{2} J_{1}{ }^{2}(\lambda)} \quad\left(\lambda=\alpha_{0, m}\right)$

$J_{2}=2 x^{-1} J_{1}-J_{0}$ 


\subsection{Double Fourier Series:}

If we have a function of two variables, then we can extend the general Fourier series as the double Fourier in the double Fourier series we can discuss two variables simultaneously. Let $f(x, y)$ a function where $x$ and $y$ are defined in the intervals $(-\pi, \pi)$ i.e. $-\pi<x<\pi$ and $-\pi<y<\pi \quad$ so we can define the general term of the double Fourier series of the function $f(x, y)$ as

$A_{i, j}=\varepsilon_{i, j}\left\{a_{i, j} \operatorname{cosix} \operatorname{cosj} y+b_{i, j} \operatorname{sinix} \operatorname{sinj} y+c_{i, j} \operatorname{cosix} \operatorname{sinj} y+d_{i, j} \operatorname{sinix} \cos j y\right\}$

$\varepsilon_{0,0}=\frac{1}{4}, \quad \varepsilon_{i, 0}=\frac{1}{2} \quad$ when $i>0$

$\varepsilon_{o, j}=\frac{1}{2} \quad$ when $j>0 \quad$ and $\varepsilon_{i, j}=1 \quad$ when $i, j>0$

And we can find the coefficients of the Double Fourier series by term integration as same as the general Fourier series with the help of below formula

$$
a_{i, j}=\frac{1}{\pi^{2}} \int_{-\pi}^{\pi} \int_{-\pi}^{\pi} f(x, y) \operatorname{cosix} \cos j y d x d y
$$

Ex: Support $f(x, y), g(x, y)$ are two real valued functions. Since they are dependent on two variables $x$, amd $y$ they shall be defined on the rectangle

$$
\begin{aligned}
& R: 0 \leq x \leq L_{1} \quad \text { and } 0 \leq y \leq L_{2} \\
& (f \mid g)=\int_{0}^{L_{1}} \int_{0}^{L_{2}} f(x, y) g(x, y) d x d y
\end{aligned}
$$

The functions $f_{m, n}$ and $g_{i, j}$ are orthogonal if 


$$
\left(f_{m, n} \mid g_{i, j}\right)=\int_{0}^{L_{1}} \int_{0}^{L_{2}} f_{m, n}(x, y) g_{i, j}(x, y) d x d y= \begin{cases}0 & \text { for } m \neq n \text { or } i \neq j \\ 1 & \text { for } m=n \text { or } i \neq j\end{cases}
$$

Complex Fourier series:

Let $f(x)$ is the given function which is defined on the interval $(-\pi, \pi)$ we know that the cos and sin functions in terms of exponential

$$
\cos \theta=\frac{e^{i \theta}+e^{-i \theta}}{2} \quad \sin \theta=\frac{e^{i \theta}+e^{-i \theta}}{2 i}
$$

So we can write the Fourier series for the given complex function as:

$$
\begin{gathered}
f(x)=\frac{a_{0}}{2}+\sum_{k=1}^{\infty}\left(a_{k} \frac{e^{i \theta}+e^{-i \theta}}{2}+b_{k} \frac{e^{i \theta}+e^{-i \theta}}{2 i}\right) \\
f(x)=\frac{a_{0}}{2}+\sum_{k=1}^{\infty} \frac{a_{k}-i b_{k}}{2} e^{i k x}+\sum_{k=1}^{\infty} \frac{a_{k}+i b_{k}}{2} e^{-i k x}=\sum_{k=-\infty}^{\infty} c k e^{i k x}
\end{gathered}
$$

We assume that notations as follows:

$$
c_{0}=\frac{a_{0}}{2}, \quad c_{k}=\frac{a_{k}-i b_{k}}{2} \quad \text { and } \quad c_{-k}=\frac{a_{k}+i b_{k}}{2}
$$

Here coefficients $c_{k}$ are called the complex Fourier coefficients and they are defined

$$
c_{k}=\frac{1}{2 \pi} \int_{-\pi}^{\pi} f(x) e^{i k x} d x \quad \text { where } k=0, \pm 1, \pm 2, \ldots \ldots
$$




\section{2-Wave Equation:}

$\frac{\partial^{2} u}{\partial t^{2}}=c^{2} \frac{\partial^{2} u}{\partial x^{2}} \quad 0<x<L, t>0$

To find $u$, we will solve this equation subject to the boundary conditions

* $u(t, 0)=0$ and $u(L, t)=0$ for all $t>0$

$$
u_{t}(0, t)=0 \text { and } u_{t}(L, t)=0 \text { for all } t>0
$$

and the initial conditions

$$
\text { * } u(x, 0)=f(x) \text { and } \frac{\partial u}{\partial t}(x, 0)=g(x) \text { for } 0<x<L
$$

Separating variables:

Step1: $u(x, t)=X(x) T(t)$

$$
\begin{gathered}
\frac{\partial^{2} u}{\partial t}=X T^{\prime \prime}, \quad \frac{\partial^{2} u}{\partial x^{2}}=X^{\prime \prime} T \\
X T^{\prime \prime}=c^{2} X^{\prime \prime} T \quad \text { then } \quad \frac{T^{\prime \prime}}{c^{2} T}=\frac{X^{\prime \prime}}{X} \\
\frac{T^{\prime \prime}}{c^{2} T}=K \quad, \frac{X^{\prime \prime}}{X}=K
\end{gathered}
$$

$K$ is an arbitrary constant called the separation constant

$X^{\prime \prime}-K X=0, \quad T^{\prime \prime}-K c^{2} T=0$

Now we get:

$X(0) T(t)=0$ and $X(L) T(t)=0$ for all $t>0$ 
if $X(0) \neq 0$ or $X(L) \neq 0$ then $T(t)$ must be 0 for all $\mathrm{t}$ and $\mathrm{u}$ is identically zero.

Then $X(0)=0$ and $X(L)=0$

$X^{\prime \prime}-K X=0, \quad X(0)=0, \quad X(L)=0$

Step2: Solving the separable equations if $K$ is positive say $K=\mu^{2}$ with $\mu>0$ then

$X^{\prime \prime}-\mu^{2} X=0$

$X(x)=c_{1} \cosh \mu x+c_{2} \sinh \mu x \quad, X(0)=0$

$0=c_{1} \cosh 0+c_{2} \sinh 0=c_{1}$

$X(x)=c_{2} \sinh \mu x \quad, X(L)=0$

$c_{2} \sinh \mu x=0$ but $\mu L \neq 0$ so $\sinh \mu L \neq 0$ and hence $c_{2}=0$ implying that $X=0$ this case $K>0$

If $K=0, X^{\prime \prime}=0$ general solution

$X(x)=c_{1} x+c_{2}$

We have to take $c_{1}=c_{2}=0$

$u=0 \quad K=-\mu^{2}<0$

The corresponding boundary value problem in $\mathrm{X}$ is

$X^{\prime \prime}-\mu^{2} X=0 \quad, X(0)=0, X(L)=0$

The general solution of the differential equation is $X=c_{1} \cos \mu x+c_{2} \sin \mu x$

The condition $X(0)=0, c_{1}=0$, hence $X=\sin \mu x$ 
The condition $X(L)=0, c_{2} \sin \mu x=0$

The trivial solution $X=0$ we take $c_{2}=1$ then $\sin \mu L=0$

$\mu=\mu_{n}=\frac{n \pi}{L}, \quad n= \pm 1, \pm 2, \ldots .$.

$X=X_{n}=\sin \frac{n \pi}{L} x, \quad n=1,2, \ldots$

We now have $K=-\mu^{2}=-\left(\frac{n \pi}{L}\right)^{2}$ and get

$$
T^{\prime \prime}+\left(c \frac{n \pi}{L}\right)^{2} T=0
$$

The general solution of this equation is

$$
\begin{gathered}
T_{n}=b_{n} \cos \lambda_{n} t+a_{n} \sin \lambda_{n} t \\
\lambda_{n}=c \frac{n \pi}{L}, \quad n=1,2, \ldots \\
u_{n}(x, t)=\sin \frac{n \pi}{L} x\left(b_{n} \cos \lambda_{n} t+a_{n} \sin \lambda_{n} t\right) \quad n=1,2 \ldots \\
u(x, t)=\sum_{n=1}^{\infty} \sin \frac{n \pi}{L} x\left(b_{n} \cos \lambda_{n} t+a_{n} \sin \lambda_{n} t\right)
\end{gathered}
$$

Step 3: Fourier series solution of the Entire problem

$u(x, 0)=f(x)=\sum_{n=1}^{\infty}\left(b_{n} \sin \frac{n \pi}{L} x\right) \quad, 0<x<L$

The series on the right is the half-range sine series expansion of $f$ 
$b_{n}=\frac{2}{L} \int_{0}^{L} f(x) \sin \frac{n \pi}{L} x d x \quad n=1,2, \ldots$

Similarly, for $a_{n}$

$g(x)=\sum_{n=1}^{\infty}\left(a_{n} \lambda_{n} \sin \frac{n \pi}{L} x\right)$

$a_{n}^{*} \lambda_{n}=\frac{2}{L} \int_{0}^{L} g(x) \sin \frac{n \pi}{L} x d x \quad n=1,2, \ldots$

Now $a_{n}^{*}=\frac{2}{c n \pi} \int_{0}^{L} g(x) \sin \frac{n \pi}{L} x d x \quad n=1,2, \ldots$

2.1 Solution of the one Dimensional Wave equation:

The solution of the one dimensional wave equation:

$$
\frac{\partial^{2} u}{\partial t^{2}}=c^{2} \frac{\partial^{2} u}{\partial x^{2}} \quad 0<x<L, t>0
$$

With boundary conditions

$u(0, t)=0 \quad$ and $u(L, t)=0 \quad$ for all $t>0$

And initial conditions

$u(x, 0)=f(x), \quad \frac{\partial u}{\partial t}(x, 0)=g(x)$ for $0<x<L$

$u(x, t)=\sum_{n=1}^{\infty} \sin \frac{n \pi}{L} x\left(b_{n} \cos \lambda_{n} t+a_{n} \sin \lambda_{n} t\right)$

Where $b_{n}=\frac{2}{L} \int_{0}^{L} f(x) \sin \frac{n \pi}{L} x d x \quad, a_{n}=\frac{2}{c n \pi} \int_{0}^{L} g(x) \sin \frac{n \pi}{L} x d x, \lambda_{n}=c \frac{n \pi}{L}, \quad n=1,2, \ldots$ 


\subsection{Heat Equation:}

The one Dimensional Heat Equation. Let $u(x, t)(0<x<L, t>0)$ represent the temperature of the point $\mathrm{x}$ of the bar at time $\mathrm{t}$. Given that the initial temperature distribution of the bar is $u(x, 0)=f(x)$

$$
\frac{\partial u}{\partial t}=c^{2} \frac{\partial^{2} u}{\partial x^{2}} \quad 0<x<L, t>0
$$

In addition, $u$ satisfies the boundary conditions $u(t, 0)=0, u(L, t)=0$ for all $t>0$

And the initial condition $u(x, 0)=f(x)$ for $0<x<L$

\section{* Separation of variables}

$u(x, t)=X(x) T(t)$ where $X(x)$ is a function of $x$ alone and $T(t)$ is a function of $t$ alone $\frac{T^{\prime}}{c^{2} T}=\frac{X^{\prime \prime}}{X}$

$\frac{T^{\prime}}{c^{2} T}=K \quad, \frac{X^{\prime \prime}}{X}=K$

$$
X^{\prime \prime}-K X=0, T^{\prime}-K c^{2} T=0 \quad \text { we get } X(0) T(t)=0, X(L) T(t)=0 \text { forall } t>0
$$$$
X(0) T(t)=0, \quad X(L)=0 \quad \text { we thus }
$$

$$
\begin{aligned}
& X^{\prime \prime}-K X=0, \quad X(0)=0, \quad X(L)=0 \quad \text { we found that } \\
& K=-\mu^{2} \quad \mu=\mu_{n}=\frac{n \pi}{L}, \quad n=1,2, \ldots \\
& X=X_{n}=\sin \frac{n \pi}{L} x, n=1,2, \ldots \\
& T^{\prime}-\left(c \frac{n \pi}{L}\right)^{2} T=0
\end{aligned}
$$




$$
\begin{aligned}
& T_{n}(t)=b_{n} e^{\lambda n^{2} t} \quad n=1,2, \ldots \\
& \lambda_{n}=\frac{n \pi}{L} \quad n=1,2, \ldots \\
& u_{n}(x, t)=b_{n} e^{-\lambda n^{2} t \sin \frac{n \pi}{L} x} \quad n=1,2, \ldots \\
& u(x, t)=\sum_{n=1}^{\infty} b_{n} e^{-\lambda n^{2} t \sin \frac{n \pi}{L} x}
\end{aligned}
$$

\subsection{Introduction to separation of variables and heat equation}

\subsubsection{Separation of variables}

This method can be used to solve many kinds of linear PDE, but as a typical example we will consider the heat equation

$$
\begin{array}{lc}
4 u_{x x}=u_{t} & 0<x<\pi, \quad t>0 \\
u(0, t)=0 & t>0 \\
u(\pi, t)=0 & t>0 \\
u(x, t)=f(x) & 0 \leq x \leq \pi
\end{array}
$$

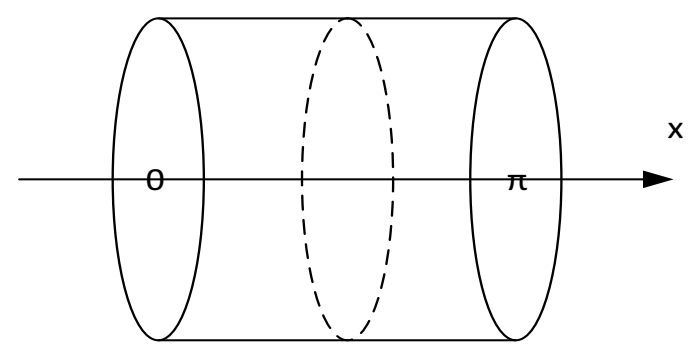

Figure 10 


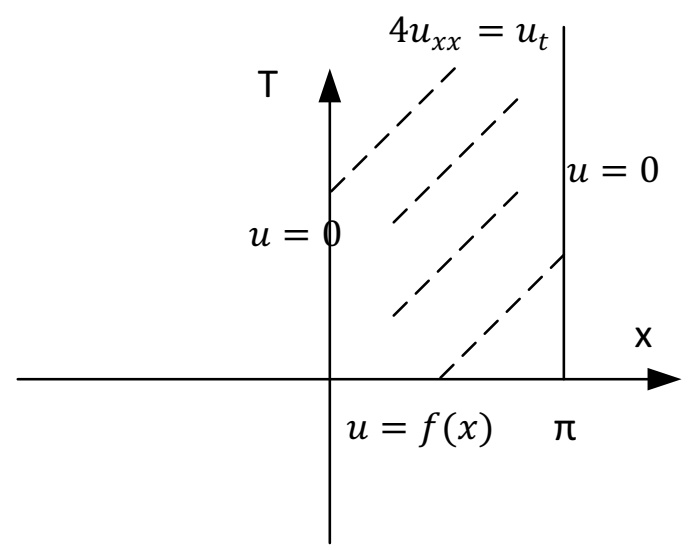

Figure 11

The idea is to assume the PDE (1) has a special form

$u(x, t)=X(x) T(t)$

Form (\#) we have $u_{x}=X^{\prime}(x) T(t)$

$u_{x x}=X^{\prime \prime}(x) T(t)$ and $u_{t}=X(x) T^{\prime}(t)$ so our PDE (1) becomes

$4 u_{x x}=u_{t} \Rightarrow 4 X^{\prime \prime}(x) T(t)=X(t) T^{\prime}(t)$

separate the variables to form

$\frac{X^{\prime \prime}(x)}{X(x)}=\frac{T^{\prime}(t)}{4 T(t)}$

Now since $L H S$ of (8) only depends on $x$ and since $R H S$ of (8) only depends on t, we must have a constant $\gamma$ such that

$$
\frac{X^{\prime \prime}}{X}=\frac{T^{\prime}}{4 T}=\gamma
$$

We form two ODEs

$T^{\prime}=4 \gamma T \quad-\rightarrow T(t)=c e^{4 \gamma T}, c$ constant

$X^{\prime \prime}-\gamma X=0-\rightarrow$ char equ $\lambda^{2}=\gamma$ thus 
$X(x)=\left\{\begin{array}{cc}A x+B, & \gamma=0 \\ A e^{\sqrt{\gamma} x}+B e^{-\sqrt{\gamma} x}, & \gamma>0 \text { \{roots are real } \pm \sqrt{\gamma}\} \\ A \cos \sqrt{-\gamma} x+B \sin \sqrt{-\gamma} x, & \gamma<0 \text { r roots are complex } \pm i \sqrt{\gamma} \text { \} }\end{array}\right.$

$u(0, t)=X(0) T(t)=0 \quad \rightarrow \quad \rightarrow X(0)=0$

$u(\pi, t)=X(\pi) T(\pi)=0 \quad \rightarrow \quad X(\pi)=0$

$T \equiv 0$ since this produces the trivial solution.

1- Case $\gamma=0 \quad X(x)=A x+B$ and $(9.1 \& 9.2)$ yield

$X(0)=A .0+B=0$ so $B=0$

$X(\pi)=A \cdot \pi+0=0 \quad$ so $A=0$

But this yields the trivial solutions.

2- Case $\gamma>0 \quad X(x)=A e^{\sqrt{\gamma} x}+B e^{-\sqrt{\gamma} x}$ and (9.1 \&9.2) yield

$X(0)=A+B=0$ so $A=-B$

$X(\pi)=A e^{\sqrt{\gamma} \pi}+B e^{-\sqrt{\gamma} \pi}=0$ we form

$A\left(e^{\sqrt{\gamma} \pi}-e^{-\sqrt{\gamma} \pi}\right)=0$ so $A=0$

But yields the trivial solution.

3- Case $\gamma<0 \quad X(x)=A \cos \sqrt{-\gamma} x+B \sin \sqrt{-\gamma} x$ and (9.1 \&9.2) yield

$X(0)=A=0$ so $A=0$

$X(\pi)=B \sin \sqrt{-\gamma} \pi=0$

But $B=0$ (trivial solution) and consider $B \sin \sqrt{-\gamma} \pi=0$ which holds when

$$
\sqrt{-\gamma} \pi=n \pi \quad n=1,2, \ldots
$$

So we have

$$
\sqrt{-\gamma}=n \quad n=1,2, \ldots
$$




$$
\begin{aligned}
& X_{a}(x)=B_{a} \sin n x \quad n=1,2, \ldots . \quad B_{n} \text { constant } \\
& T(t)=c e^{4 \gamma t} \text { to form } \\
& T_{n}(t)=c_{n} e^{-4 a^{2} t} \quad n=1,2, \ldots . \quad c_{n} \text { constant }
\end{aligned}
$$

From our assumed form $u(x, t)=X(x) T(t)$, we form a sequence

$$
\begin{aligned}
& u_{n}(x, t)=X_{n}(x) T_{n}(t) \quad n=1,2, \ldots . . \\
& u_{n}(x, t)=\left(B_{a} \sin n x\right)\left(c_{n} e^{-4 n^{2} t}\right)=b_{n} e^{-4 n^{2} t} \sin n x \quad\left(b_{n}=B_{n} c_{n}\right)
\end{aligned}
$$

Since (4), (5) and (6) are linear thomog every sum of solution is also a solv, hence we can form

$$
u(x, t)=\sum_{n=1}^{\infty} u_{n}(x, t)=\sum_{n=1}^{\infty} b_{n} e^{-4 n^{2} t} \sin n x
$$

To define $b_{n}$ we use

$$
u(x, 0)=f(x)=\sum_{n=1}^{\infty} b_{n} \sin n x
$$

\subsection{How we use Fourier series:}

We can write our initial temperature $u(x, 0)=f(x)$ as an infinite sum involving $\sin n x$ then we may calculate the $b_{n}$ and hence determine the exact solution to the problem.

If $u(x, 0)=f(x)=\sin x-2 \sin 3 x$ then we may write it as

$u(x, 0)=f(x)=\sin x-2 \sin 3 x=\sum_{n=1}^{\infty} b_{n} \sin n x=b_{1} \sin x+b_{2} \sin 2 x+b_{3} \sin 3 x+\cdots$

And equating the coefficients we see that $b_{1}=1, b_{3}=-2$ and all the other $b_{i}$ must be zero, thus our solution $\sum_{n=1}^{\infty} b_{n} e^{-4 n^{2} t} \sin n x$

$$
u(x, t)=e^{-4 t} \sin x-2 e^{-36 t} \sin 3 x
$$


However, if we had an initial temperature function of the form $u(x, 0)=f(x)=X$ then to determine the $b_{n}$ we would consider

$$
u(x, 0)=f(x)=X=\sum_{n=1}^{\infty} b_{n} \sin n x
$$

This question leads Fourier to develop a type of series known as Fourier series involving sin and $\cos$.

The $b_{n}$ can be calculated from $b_{n}=\frac{2}{\pi} \int_{0}^{\pi} f(x) \sin n x d x$

Bar with insulated ends

\section{Ex: consider the boundary value problem}

$u_{t}=u_{x x} \quad 0<x<L \quad t>0$

$u_{x}(0, t)=0$

$u_{x}(L, t)=0$

$t>0$

$u(X, 0)=h(x 0=X(L-x) \quad 0 \leq x \leq L$

This determines the temperature distribution $u(x, t)$ in a rod of length $\mathrm{L}$ insulated laterally and at both ends $X=0, X=L$ and with the initial temperature $h(x)$. Assume solv $u$ to (1) is of form $u(x, t)=X(t) T(t)$ we have

$u_{x x}(x, t)=X^{\prime \prime}(x) T(t) \quad, \quad u_{t}(x, t)=X(x) T^{\prime}(t)$

Hence (11) becomes $X(x) T^{\prime}(t)=X^{\prime \prime}(x) T(t)$ and we separate the variables to form

$\frac{X^{\prime \prime}(x)}{X(x)}=\frac{T^{\prime}(t)}{T(t)}=\gamma$

Where $\gamma$ is the "separation constant" we form two ODEs 
$T^{\prime}=\gamma T \rightarrow T(t)=c e^{\gamma t}$

$X^{\prime \prime}-\gamma X=0$, we form the cherecve $\lambda^{2}=\gamma$ Thus:

$X(x)= \begin{cases}A x+B, & \gamma=0 \\ A e^{\sqrt{\gamma} x}+B e^{-\sqrt{\gamma} x}, & \gamma>0 \\ A \cos \sqrt{-\gamma} x+B \sin \sqrt{-\gamma} x, & \gamma<0\end{cases}$

Now (12) and $u=X . T$ yields

$\begin{array}{ll}0=u_{x}(0, t)=X^{\prime}(0) T(t) \rightarrow & X^{\prime}(0)=0 \\ 0=u_{x}(L, t)=X^{\prime}(L) T(t) \rightarrow & X^{\prime}(L)=0\end{array}$

1- Case $\gamma=0, A=0$ and $B$ arbitrary so $X \equiv B$

2- Case $\gamma>0, A \sqrt{\gamma}-B \sqrt{\gamma}=0 \Rightarrow A=B$

$A \sqrt{\gamma} e^{\sqrt{\gamma L}}-B \sqrt{\gamma} e^{-\sqrt{\gamma L}}=0$ so we form

$A \sqrt{\gamma}\left[e^{\sqrt{\gamma L}}-\frac{1}{e^{\sqrt{\gamma L}}}\right] \Rightarrow A=0$ so $B=0$ Trival case

3- Case $\gamma<0, \quad-\sqrt{-\gamma} A \sin 0+\sqrt{-\gamma} B \cos 0=0$

$-\sqrt{-\gamma} A \sin \sqrt{-\gamma} L+\sqrt{-\gamma} B \cos \sqrt{-\gamma} L=0$

And we have $\sin \sqrt{-\gamma} L=0$ which has solution $\sqrt{-\gamma} L=n \pi \quad n=1,2, \ldots$

And hence $\sqrt{-\gamma}=\frac{n \pi}{L} \quad n=1,2, \ldots$

Thus we for a sequence of functions

$$
X_{n}(x)=A_{n} \cos \frac{n \pi x}{L} \quad n=1,2, \ldots
$$

And

$$
T_{n}(t)=c_{n} e^{-\left(\frac{n \pi}{L}\right)^{2} t} \quad n=1,2, \ldots
$$




$$
\begin{gathered}
\text { Hence } u_{n}(x, t)=X_{n}(x) T_{n}(t) \quad n=1,2, \ldots \\
u(x, t)=\sum_{n=1}^{\infty} u_{n}(x, t)=a_{0}+\sum_{n=1}^{\infty} u_{n}(x, t)=a_{0}+\sum_{n=1}^{\infty} a_{n} \cos \left(\frac{n \pi x}{L}\right) e^{-\left(\frac{n \pi}{L}\right)^{2} t}
\end{gathered}
$$

Now (13) yields $u(x, 0)=h(x)==a_{0}+\sum_{n=1}^{\infty} a_{n} \cos \left(\frac{2 \pi x}{2}\right)$

Now this is just a Fourier cosine series for $h$ this

$$
\begin{aligned}
& a_{0}=\frac{1}{L} \int_{0}^{L} h(x) d x \\
& a_{n}=\frac{2}{L} \int_{0}^{L} h(x) \cos \frac{n \pi x}{L} d x \\
& a_{0}=\frac{1}{L} \int_{0}^{L} x(L-x) d x=\frac{1}{L} \int_{0}^{L}\left(L x-x^{2}\right) d x=\frac{1}{L}\left[\frac{L}{2} x^{2}-\frac{1}{3} x^{3}\right]_{0}^{L}=\frac{L^{2}}{6}
\end{aligned}
$$

Since out temperature decays exponentially in the summoned we have

$$
u(x, t)_{t \rightarrow \infty} \rightarrow a_{0}=\frac{L^{2}}{6}
$$

\subsection{The two Dimensional Wave and Heat Equations:}

Two dimensional wave equation is

$\frac{\partial^{2} u}{\partial t^{2}}=c^{2}\left(\frac{\partial^{2} u}{\partial x^{2}}+\frac{\partial^{2} u}{\partial y^{2}}\right) \quad 0<x<a, \quad 0<y<b, \quad t>0$

Where $u=u(x, y, t)$ at the point $(x, y)$ at time $t$

We have $u=u(x, y, t)=0$ on the boundary for all $t \geq 0$

$$
u(0, y, t)=0, u(a, y, t)=0 \text { for } 0 \leq y \leq b \text { and } t \geq 0
$$


The initial conditions are

$$
\begin{aligned}
& u(x, y, 0)=f(x, y) \text { and } \frac{\partial u}{\partial t}(x, y, 0)=g(x, y) \\
& u(x, y, t)=X(x) Y(y) T(t) \\
& X Y T^{\prime \prime}=c^{2}\left(X^{\prime \prime} Y T+X Y^{\prime \prime} T\right) \\
& \frac{T^{\prime \prime}}{c^{2} T}=\frac{X^{\prime \prime}}{X}+\frac{Y^{\prime \prime}}{Y} \\
& \frac{T^{\prime \prime}}{c^{2} T}=-K^{2} \text { and } \frac{X^{\prime \prime}}{X}+\frac{Y^{\prime \prime}}{Y}=-K^{2}, \quad K>0 \\
& T^{\prime \prime}+K^{2} c^{2} T=0 \\
& \frac{X^{\prime \prime}}{X}=\frac{Y^{\prime \prime}}{Y}-K^{2} \\
& \frac{X^{\prime \prime}}{X}=-\mu^{2}, \quad \frac{Y^{\prime \prime}}{Y}-K^{2}=-\mu^{2} \quad \mu>0 \\
& X^{\prime \prime}+\mu^{2} X=0, \quad Y^{\prime \prime}+V^{2} Y=0, \quad V^{2}=K^{2}-\mu^{2} \\
& X^{\prime \prime}+\mu^{2} X=0, \quad X(0)=0, \quad X(a)=0 \\
& Y^{\prime \prime}+V^{2} Y=0, \quad Y(0)=0, \quad Y(b)=0 \\
& T^{\prime \prime}+c^{2} K^{2} T=0 \quad K^{2}=V^{2}+\mu^{2} \\
& \text { 2.6 Solution of the separated Equations: } \\
& X(x)=c_{1} \cos \mu x+c_{2} \sin \mu x \\
& Y(y)=d \cos V x+d_{2} \sin V x \\
& T(t)=e_{1} \cos c K t+e_{2} \sin c K t \quad\left(K^{2}=V^{2}+\mu^{2}\right)
\end{aligned}
$$


We get $c_{1}=0$ and $c_{2} \sin \mu a=0$

$d_{1}=0$ and $d \sin V a=0$ thus

$\mu=\mu_{m}=\frac{m \pi}{a} \quad$ and $V=V_{n}=\frac{m \pi}{b} \quad m, n=1,2, \ldots$

So $X_{m}(x)=\sin \frac{m \pi}{a} x, \quad Y_{n}(y)=\sin \frac{m \pi}{b} y$

If $m=0$ or $n=0$ the solutions are identically zero.

For $m, n=1,2, \ldots$ we have

$K=K_{m n}=\sqrt{\mu_{m n}^{2}}=\sqrt{\mu_{m}^{2}+V_{n}^{2}}=\sqrt{\frac{m^{2} \pi^{2}}{a^{2}}+\frac{n^{2} \pi^{2}}{b^{2}}}$

$T(t)=T_{m n}(t)=B_{m n} \cos \lambda_{m n} t+C_{m n} \sin \lambda_{m n} t, \quad \lambda_{m n}=c \pi \sqrt{\frac{m^{2}}{a^{2}}+\frac{n^{2}}{b^{2}}}$

$u_{m n}(x, y, t)=\sin \frac{m \pi}{a} x \sin \frac{n \pi}{b} y\left(B_{m n} \cos \lambda_{m n} t+C_{m n} \sin \lambda_{m n} t\right)$

The function $u_{m n}$ is called the normal modes of the two dimensional wave equation.

\section{3- Double Fourier series solution of the entire problem:}

$u(x, y, t)=\sum_{n=1}^{\infty} \sum_{m=1}^{\infty}\left(B_{m n} \cos \lambda_{m n} t+C_{m n} \sin \lambda_{m n} t\right) \sin \frac{m \pi}{a} x \sin \frac{n \pi}{b} y$

From the initial condition $u(x, y, o)=f(x, y)$ we get 


$$
\begin{aligned}
f(x, y)=\sum_{n=1}^{\infty} \sum_{m=1}^{\infty} B_{m n} \sin \frac{m \pi}{a} x \sin \frac{n \pi}{b} y \\
\quad=\int_{0}^{b} \int_{0}^{a} \sin \frac{m \pi}{a} x \sin \frac{m \pi}{a} x \sin \frac{n \pi}{b} y \sin \frac{m^{\prime} \pi}{a} x \sin \frac{n^{\prime} \pi}{b} y d x d y=0
\end{aligned}
$$

If $(m, n) \neq\left(m^{\prime}, n^{\prime}\right)$ also if $(m, n)=\left(m^{\prime}, n^{\prime}\right)$ then we get

$\int_{0}^{b} \int_{0}^{a} \sin ^{2} \frac{m \pi}{a} x \sin ^{2} \frac{n \pi}{b} y d x d y=\frac{a b}{4}$

$B_{m n}=\frac{4}{a b} \int_{0}^{b} \int_{0}^{a} f(x, y) \sin \frac{m \pi}{a} x \sin \frac{n \pi}{b} y d x d y$ this is called the double Fourier series of $f$, we get $g(x, y)=\sum_{n=1}^{\infty} \sum_{m=1}^{\infty} C_{m n} \lambda_{m n} \sin \frac{m \pi}{a} x \sin \frac{n \pi}{b} y$

$C_{m n}=\frac{4}{a b \lambda_{m n}} \int_{0}^{b} \int_{0}^{a} g(x, y) \sin \frac{m \pi}{a} x \sin \frac{n \pi}{b} y d x d y$

\subsection{The solution of the two Dimensional wave equation}

$u(x, y, t)=\sum_{n=1}^{\infty} \sum_{m=1}^{\infty}\left(B_{m n} \cos \lambda_{m n} t+C_{m n} \sin \lambda_{m n} t\right) \sin \frac{m \pi}{a} x \sin \frac{n \pi}{b} y$

Where $\lambda_{m n}=c \pi \sqrt{\frac{m^{2}}{a^{2}}+\frac{n^{2}}{b^{2}}}$

\subsection{Solution of the two Dimensional Heat equation for a Rectangle:}

$\frac{\partial u}{\partial t}=c^{2}\left(\frac{\partial^{2} u}{\partial x^{2}}+\frac{\partial^{2} u}{\partial y^{2}}\right) \quad 0<x<a, \quad 0<y<b, \quad t>0$

With boundary conditions

$$
\begin{aligned}
& u(0, y, t)=u(a, y, t)=0, \text { for } 0<y<b, \quad t>0 \\
& u(x, 0, t)=u(x, b, t)=0, \text { for } 0<x<a, \quad t>0
\end{aligned}
$$


And initial condition $u(x, y, 0) f(x, y) \quad 0<x<a, 0<y<b$

$(x, y, t)=\sum_{n=1}^{\infty} \sum_{m=1}^{\infty}\left(A_{m n} \sin \frac{m \pi}{a} x \sin \frac{n \pi}{b} y e^{-\lambda^{2} m n t}\right.$

Where $\lambda_{m n}=c \pi \sqrt{\frac{m^{2}}{a^{2}}+\frac{n^{2}}{b^{2}}}$

$A_{m n}=\frac{4}{a b} \int_{0}^{b} \int_{0}^{a} f(x, y) \sin \frac{m \pi}{a} x \sin \frac{n \pi}{b} y d x d y \quad m, n=1,2, \ldots$

3-3 Laplace's Equation in Rectangular coordinates:

$\frac{\partial u}{\partial t}=c^{2}\left(\frac{\partial^{2} u}{\partial x^{2}}\right) \quad 0<x<L, \quad t>0$

$u_{x x}=0 \quad, u(x)=c_{1} x+c_{2}$

$\frac{\partial^{2} u}{\partial x^{2}}+\frac{\partial^{2} u}{\partial y^{2}}=0 \quad 0<x<a, 0<y<b$

Now $u(x, 0)=f_{1}(x), \quad u(x, b)=f_{2}(x) \quad 0<x<a$

$$
u(0, y)=g_{1}(y), \quad u(a, y)=g_{2}(x) \quad 0<y<b
$$

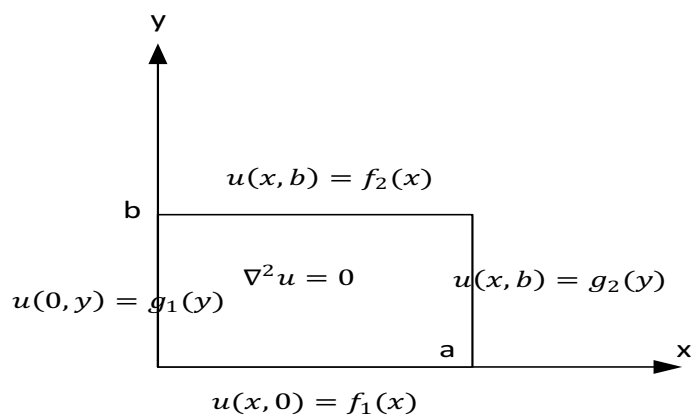


$\underline{E x}: X^{\prime \prime}+K X=0, Y^{\prime \prime}+K Y=0 \quad K$ is the separation constant

$X(0)=0, \quad X(a)=0, \quad Y(0)=0$

$K \leq 0, \quad K=\mu^{2}>0$

$X=c_{1} \cos \mu x+c_{2} \sin \mu x$

$c_{1}=0 \quad \mu=\mu_{n}=\frac{n \pi}{a} \quad n=1,2, \ldots$

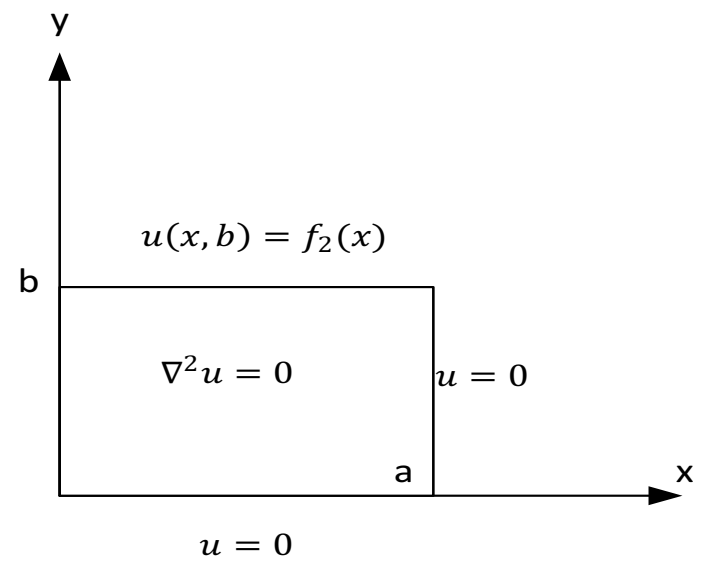

Figure 13

Hence $X_{n}(x)=\sin \frac{n \pi}{a} x, \quad n=1,2, \ldots$

Now to $\mathrm{Y}$ with $K=\mu^{2}{ }_{n}$ we have

$Y=A_{n} \cosh \mu_{n} y+B_{n} \sinh \mu_{n} y$

$Y(0)=0$ we find that $A_{n}=0$

Hence $\quad Y_{n}=B_{n} \sinh \mu_{n} y$

We get the general form of the solution: 


$$
\begin{aligned}
& u(x, y)=\sum_{n=1}^{\infty} B_{n} \sin \frac{n \pi}{a} x \sinh \frac{n \pi}{a} y \\
& u(x, b)=f_{2}(x) \\
& f_{2}(x)=\sum_{n=1}^{\infty} B_{n} \sinh \frac{n \pi b}{a} \sin \frac{n \pi}{a} x \\
& B_{n}=\frac{2}{a \sinh \frac{n \pi b}{a}} \int_{0}^{a} f_{2}(x) \sin \frac{n \pi}{a} x d x \quad n=1,2, \ldots \\
& D_{n}=\frac{2}{b \sinh \frac{n \pi a}{b}} \int_{0}^{b} g_{2}(y) \sin \frac{n \pi}{b} y d y \quad n=1,2, \ldots \\
& A_{n}=\frac{2}{a \sinh \frac{n \pi b}{a}} \int_{0}^{a} f_{1}(x) \sin \frac{n \pi}{a} x d x \quad n=1,2, \ldots \\
& C_{n}=\frac{2}{b \sinh \frac{n \pi a}{b}} \int_{0}^{b} g_{1}(y) \sin \frac{n \pi}{b} y d y \quad n=1,2, \ldots
\end{aligned}
$$

Solution of the Dirichlet problem in a rectangle:

The solution of the two dimensional Dirichlet problem in figure is

$$
\begin{aligned}
u(x, y)=\sum_{n=1}^{\infty} A_{n} \sin \frac{n \pi}{a} x \sinh \frac{n \pi}{a}(b-y)+\sum_{n=1}^{\infty} B_{n} \sin \frac{n \pi}{a} x \sinh \frac{n \pi}{a} y \\
\quad+\sum_{n=1}^{\infty} C_{n} \sinh \frac{n \pi}{b}(a-x) \sin \frac{n \pi}{b} y+\sum_{n=1}^{\infty} D_{n} \sinh \frac{n \pi}{b} x \sin \frac{n \pi}{b} y
\end{aligned}
$$




\section{4-References}

1- Differential Equations and Boundary Value Problems (Computing and Modeling), Fifth Edition, C. Henry Edward, David E. Penny, David T. Calvis, October 2014

2- Advanced Engineering Mathematics 10th edition, Erwin Kreyszig August 2011

3- Partial Differential Equations with Fourier Series and Boundary Value Problem, second Edition, Nakhle H. Asmar, 2004

4- Elementary Differential Equaions and Boundary Value Problems, $10^{\text {th }}$ Edition, William E.. Boyce, Richard C. DiPrima, October 2012. 